\title{
Transforming two-dimensional guided light using nonmagnetic metamaterial waveguides
}

\author{
Sophie Viaene, ${ }^{1,2}$ Vincent Ginis, ${ }^{1}$ Jan Danckaert, ${ }^{1}$ and Philippe Tassin ${ }^{2,1, *}$ \\ ${ }^{1}$ Applied Physics Research Group, Vrije Universiteit Brussel, Pleinlaan 2, B-1050 Brussel, Belgium \\ ${ }^{2}$ Department of Physics, Chalmers University of Technology, SE-412 96 Göteborg, Sweden \\ (Received 30 September 2015; revised manuscript received 27 January 2016; published 19 February 2016)
}

\begin{abstract}
Almost a decade ago, transformation optics established a geometrical perspective to describe the interaction of light with structured matter, enhancing our understanding and control of light. However, despite their huge technological relevance in applications such as optical circuitry, optical detection, and actuation, guided electromagnetic waves along dielectric waveguides have not yet benefited from the flexibility and conceptual simplicity of transformation optics. Indeed, transformation optics inherently imposes metamaterials not only inside the waveguide's core but also in the surrounding substrate and cladding. Here we restore the two-dimensional nature of guided electromagnetic waves by introducing a thickness variation on an anisotropic dielectric core according to alternative two-dimensional equivalence relations. Our waveguides require metamaterials only inside the core with the additional advantage that the metamaterials need not be magnetic and, hence, our purely dielectric waveguides are low loss. We verify the versatility of our theory with full wave simulations of three crucial functionalities: beam bending, beam splitting, and lensing. Our method opens up the toolbox of transformation optics to a plethora of waveguide-based devices.
\end{abstract}

DOI: 10.1103/PhysRevB.93.085429

Geometrical reasoning played a crucial role in the historical development of optics as a scientific discipline, and its successes are associated with the names of great scientists like Snell, de Fermat, Huygens, Newton, and others. To this day, the design of many optical components, e.g., microscopes, displays, and fibers, is based on the ray picture of light, valid for electromagnetic waves inside media with slowly varying refractive index distributions [1]. Through the advent of metamaterials and photonic crystals [2-8] - artificial materials whose electromagnetic properties are determined by subwavelength unit cells_-light may be manipulated by enhanced optical properties at the micro- and nanoscale. As a result, there is a growing need for analytical tools to model and design metamaterial devices that act upon the electric and magnetic components of light [9].

With the design and experimental demonstration of invisibility cloaks [10-14], transformation optics proved to be an adequate geometrical tool to explore the full potential of metamaterials. Succinctly, transformation optics relies on the form invariance of Maxwell's equations to determine appropriate material properties, i.e., permittivity and permeability distributions, that materialize unconventional light flows based on the deformation of a coordinate system. Using this geometrical perspective of the interaction of light with structured matter, researchers have discovered and reconsidered many optical phenomena in three-dimensional metamaterials with regard to wave propagation [15-17], subwavelength sensing [18], Cherenkov radiation [19], effective gauges [20], and many more.

To enhance control on the propagation of surface waves confined to a single interface [21-23], several research groups successfully applied the existing framework of transformation optics to surface waves along graphene-dielectric [24,25] and metal-dielectric interfaces [26-30]. Indeed, at frequencies far away from the surface plasmon resonance of a metal-dielectric

${ }^{*}$ Corresponding author. interface, the evanescent tails of the surface plasmons extend substantially into the dielectric material. As a result, surface plasmons can be made to follow coordinate-based trajectories if the dielectric is replaced by a metamaterial according to the conventional recipe of transformation optics. Unfortunately, the propagation range of surface plasmon polaritons is severely limited by dissipative loss in the metallic substrate [31,32], especially at infrared and optical frequencies. Therefore, although a simple metal-dielectric interface is amenable to transformation optics, it is not an ideal platform for long-range processing of surface waves.

In this contribution, we introduce a radically different formulation of transformation optics applicable to electromagnetic waves confined to a purely dielectric slab waveguide. The resulting low-loss metamaterial waveguides can mold the flow of light in optical circuitry, optical detection, and actuation applications [33-35]. In the first part of this contribution, we apply a two-dimensional conformal coordinate transformation to the symmetry plane of a slab waveguide [36]. These twodimensional transformations are naturally compatible with a planar waveguide structure and automatically lead to nonmagnetic metamaterial implementations according to our alternative equivalence relations. In the second part of this contribution, we discuss the effectiveness and versatility of our equivalence relations with three proof-of-concept waveguide components: a beam bender, a beam splitter, and a conformal lens.

\section{TRANSFORMING TWO-DIMENSIONAL GUIDED MODES}

The main obstruction for the application of the conventional framework of transformation optics is the following: in order to implement a two-dimensional coordinate transformation of light flows along the symmetry plane of the waveguide [Figs. 1(a)-1(c)], the transformation optics recipe imposes a bulky, magnetic, and lossy three-dimensional material of infinite extent. Indeed, because the transformation is insensitive to the spatial coordinate perpendicular to the 
(a)

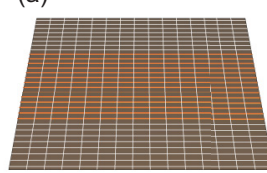

$(u, v)$

(d)

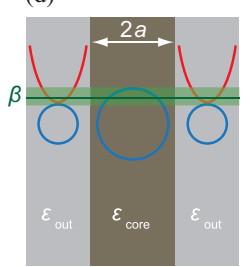

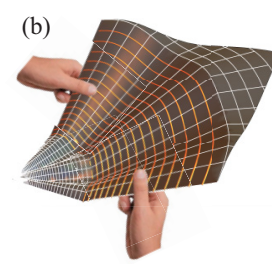

(e)

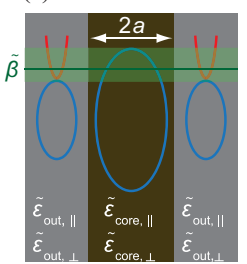

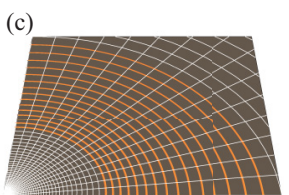

$(x, y)$ (f)

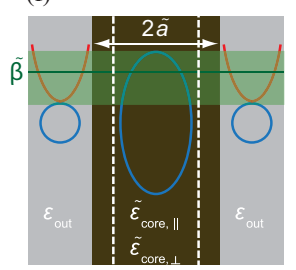

FIG. 1. Our design uses a nonmagnetic uniaxial metamaterial waveguide of varying thickness to impose two-dimensional flows of light. (a) The symmetry plane of a slab waveguide is locally stretched by a two-dimensional conformal coordinate transformation (b) so that light bends over $90^{\circ}$ as if it experiences a geometry $\gamma(x, y)$ (c). (d) Accordingly, the vector space of the incident guided mode with propagation constant $\beta$ (green) is stretched in the symmetry plane (e). The total wave vectors lie respectively on elliptical (hyperbolic) isofrequency contours of the wave equation in blue (red) inside the core (outer) region. According to the traditional recipe of transformation optics, the exponential tails $\left(k_{1}\right)$ and thickness $a$ are preserved because metamaterials are implemented in the core and outer layers. (f) To preserve confinement and to impose a globally stretched propagation vector without metamaterials in the outer layers, a thickness variation $\tilde{a}$ ensures the continuity conditions at the interfaces at the expense of changes in the exponential tails $\left(k_{1}\right)$.

waveguide, the material implementation of transformation optics is also independent of this coordinate. Moreover, the conventional application of transformation optics requires impedance-matched magnetic metamaterials whose implementation is inherently lossy [37,38]. Here, we demonstrate that in the case of two-dimensional conformal transformations, the transformed guided light flows can be materialized inside nonmagnetic metamaterial cores of varying thickness without the need for material implementations in the surrounding regions. To this aim, we introduce a set of two-dimensional equivalence relations.

Our analysis starts from the consideration that-instead of the full Maxwell equations - only those equations that govern guided waves are required to construct a two-dimensional framework. In particular, the transverse-magnetic guided modes of a slab waveguide with thickness $2 a$ and dielectric profile $\epsilon(z)$ - consisting of a high-index core layer $\epsilon_{\text {core }}$ and low-index outer layers $\epsilon_{\text {out }}$-are determined by two scalar equations: the Helmholtz equation, which governs the in-plane propagation along the waveguide, and the dispersion relation, which imposes the continuity of the confined mode profile at the material interfaces. This concept is illustrated in Fig. 1, by looking at our design process in the spatial [Figs. 1(a)-1(c)] and reciprocal space [Figs. 1(d)-1(f)] of a guided mode.

For the initial isotropic, homogeneous waveguide [Fig. 1(d)], the reciprocal space is completely determined by the propagation constant $\beta$ along the waveguide symmetry plane and the angular frequency $\omega$. Indeed, the guided

mode consists of a fixed confined transverse profile that is characterized by a transversal wave vector component $k_{2}$ inside the core region and an exponential decay with extinction coefficient $k_{1}$ in the surrounding layers. These are defined in terms of $\beta, \omega$ and the dielectric profile $\epsilon(z)$ by the Helmholtz wave equation of the in-plane magnetic field

$$
\left[\Delta_{x y} \pm k_{1,2}^{2}+\epsilon_{\text {out }, \text { core }} \frac{\omega^{2}}{c^{2}}\right] H_{\|}=0,
$$

where $\Delta_{x y}$ is the Laplacian associated with the waveguide symmetry plane and the plus (minus) sign relates to $k_{1}\left(k_{2}\right)$. In other words, the Helmholtz equation [Eq. (1)] constrains the total wave vectors $\left(\beta, k_{1,2}\right)$ to a hyperbolic isofrequency contour in the surrounding regions (red) and to an ellipsoidal isofrequency contour (blue) inside the core. Although both contours are compatible for a range of propagation constants $\beta$ in the green band, only one specific propagation constant $\beta$ of the incident mode - indicated by the green line-allows for a continuous mode profile at the material interfaces $z=$ $\pm a$. Mathematically, the selected propagation constant $\beta$ of a transverse magnetic fundamental mode satisfies the following dispersion relation:

$$
\tan \left[k_{2}(\omega, \beta) a\right]=\frac{\epsilon_{\text {core }} k_{1}(\omega, \beta)}{\epsilon_{\text {out }} k_{2}(\omega, \beta)} .
$$

To motivate our equivalence relations, we now consider the effects of a two-dimensional conformal transformation on the incident mode [Fig. 1(e)]. In the Supplemental Material [39], we show that the Helmholtz wave equation, describing the propagation along the waveguide with induced geometry $\gamma(x, y)$, is materialized by a nonmagnetic, uniaxial material $\tilde{\epsilon}_{\perp}=\gamma(x, y) \epsilon(z), \tilde{\epsilon}_{\|}=\epsilon(z)$. To address the transformation in reciprocal space, we emphasize that each two-dimensional conformal transformation locally reduces to a constant stretching $X$ of the symmetry plane. As a consequence, the transformation locally stretches the in-plane propagation vector of the guided mode $\tilde{\beta}=X \beta$ and preserves the transverse mode profiles characterized by the variables $k_{1}$ and $k_{2}$. According to the conventional three-dimensional equivalence relations of transformation optics, this global deformation of the in-plane wave vector needs to be imposed by nontrivial materials both inside the core and the surrounding regions of the waveguide, leading to an inconvenient bulky design.

We now introduce the main idea of this paper: by modification of the core region's thickness, we preserve the transformed in-plane solutions-identified by the in-plane propagation constant $\tilde{\beta}$-without material implementations in the cladding and substrate regions [Fig. 1(f)]. In other words, we only implement the nonmagnetic, uniaxial medium inside the core region, to preserve both the transverse wave vector $k_{2}$ and the desired propagation constant $\tilde{\beta}$, and we allow for changes in the extinction coefficient $k_{1}^{2}=\gamma(x, y) \beta^{2}-\epsilon_{\text {out }}\left(\omega^{2} / c^{2}\right)$. In accordance with Eq. (2), the continuity of the fields at the interfaces is restored by a variation of the thickness $\tilde{a}$ [Fig. 1(f)],

$$
\tilde{a}=\frac{1}{k_{2}} \arctan \left(\frac{\epsilon_{\mathrm{core}} \sqrt{\gamma(x, y) \beta^{2}-\epsilon_{\mathrm{out}} \frac{\omega^{2}}{c^{2}}}}{\epsilon_{\text {out }} k_{2}}\right),
$$


(a)

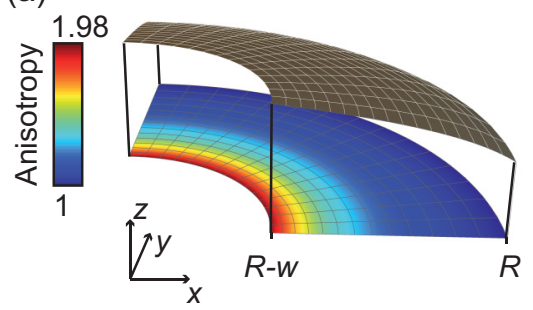

(b)

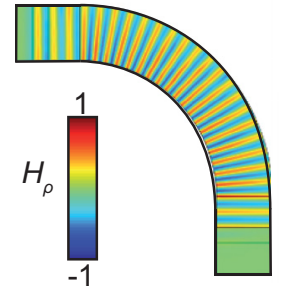

(c) (d)

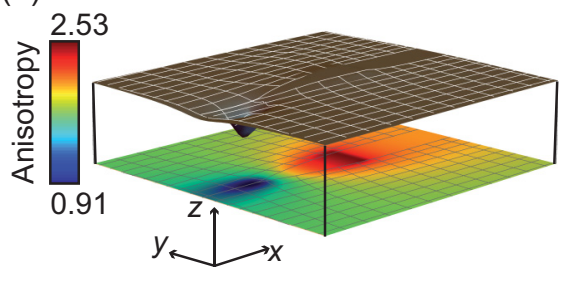

(e)

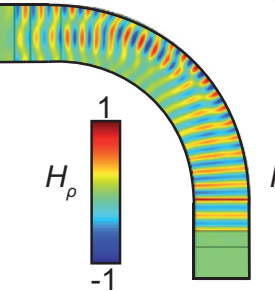

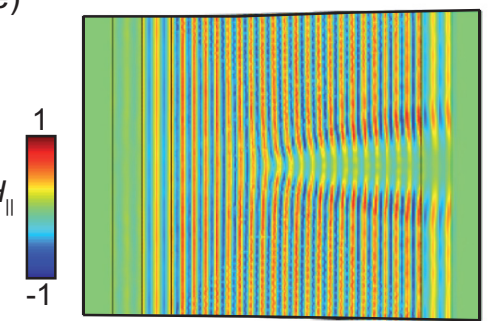

(f)

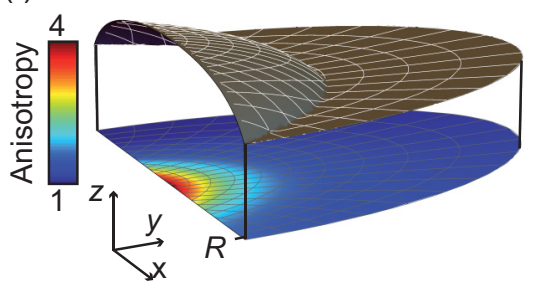

(g)

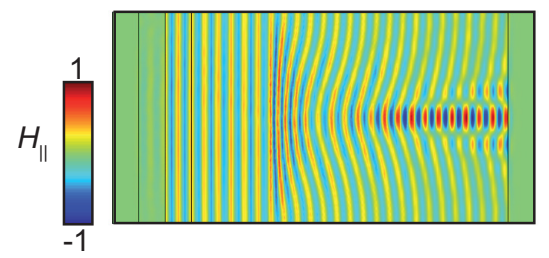

FIG. 2. Demonstration and numerical verification of the versatility of the two-dimensional equivalence relations for a beam bender (a)-(c), beam splitter (d)-(e), and Möbius lens (f)-(g). In all these examples, both the anisotropy (visualized by surface coloring on the symmetry plane of the waveguide) and the thickness variations of the core medium (visualized by the height of the floating surface representing the upper interface $z=\tilde{a}$ ) manipulate the in-plane magnetic fields in a desired way and correspond to technologically feasible parameters. Without thickness variations (c), the in-plane magnetic fields cannot complete the bend.

which preserves the initial dispersion $(\beta, \omega)$ despite the modified extinction coefficient. The transformed wave equation and the continuity of the tangential fields are thus imposed by the anisotropic permittivity and the thickness variation of the core, respectively.

\section{NONMAGNETIC METAMATERIAL WAVEGUIDES}

In the second part of this paper, we illustrate the versatility and effectiveness of our nonmagnetic equivalence relations using three conformal transformations: an exponential map implementing a beam bender [Figs. 2(a)-2(c)], a curvefactor Schwarz-Christoffel transformation implementing a beam splitter [Figs. 2(d)-2(e)], and a Möbius transformation implementing a lens [Figs. 2(f) $-2(\mathrm{~g})]$. For each of these examples we have visualized the upper half of our symmetric waveguide, representing the anisotropy of the metamaterial cores with a surface coloring on the waveguide symmetry plane and the thickness variation of the upper interface at $z=\tilde{a}$ with a floating surface. Furthermore, we note that conformal transformations have a rich history in physics and engineering [40,41]; e.g., they also contributed to several three-dimensional transformation-optical devices [16], so that a variety of two-dimensional conformal transformations are available from literature.

As a first example we use a logarithmic map [16] to design a beam bender [Figs. 2(a)-2(c)]. This two-dimensional implementation requires modest thickness variations and anisotropies, illustrated for a beamwidth $w=24 a$ and outer radius $R=82.7 a$ in terms of the initial thickness $2 a=$ $0.4 \mu \mathrm{m}$. Qualitatively, comparisons of in-plane magnetic fields (Fig. 2(b) and Figs. S4-S7 of the Supplemental Material [39]) to the conventional transformation-optics implementation confirm that the combined variation of the anisotropy $\left(\epsilon_{\perp} / \epsilon_{\|}\right)=$ $\gamma(x, y)$ in the core and the thickness $\tilde{a}(x, y)$ lead to efficient beam bends, although eventually the size of such a beam bend will be limited by the Miller limit [42]. Moreover, as is shown by Fig. 2(c), anisotropic beam bends without thickness variations cannot preserve a global propagation constant due to incompatible continuity conditions. Therefore, guided waves cannot propagate without thickness variations.

To test the equivalence in a quantitative way, we determine the throughput while reducing the outer radius $R$ of the bends at a fixed initial width $w$ (Fig. 3). In this way, the anisotropy of the core $\left(\epsilon_{\perp} / \epsilon_{\|}\right)$increases with the benchmark $(w / R)$. Impressively, these throughputs range from $84 \%$ to 93\% for microscale inner radii between $3.26 \mu \mathrm{m}$ and $25.9 \mu \mathrm{m}$, comparable to designs by three-dimensional transformation optics $(86 \%$ to $95 \%)$. However, when using an isotropic medium $\tilde{\epsilon}_{\text {core }}=\gamma(x, y) \epsilon_{\text {core }}$ together with the required thickness variations, the throughputs fall considerably at radii close to the effective incident wavelength $\lambda=1.5 \mu \mathrm{m}$, leading to poor beam benders (Fig. 3 and Fig. S6). Indeed, isotropic media do not preserve the incident polarization of the guided mode so that the thickness variation only partially applies, i.e., only to the transverse-magnetic part of the light. Thus, we have established that anisotropy and thickness variations are both indispensable to our equivalence on a qualitative and quantitative level.

As a second example, we design a beam splitter in Figs. 2(d)-2(e) that relies on a curve-factor SchwarzChristoffel transformation [41]. The coordinate lines and light flows follow the outline of a polygon with parametrized corners and curved boundaries specified by the curve factor [Figs. 2(d)]. Our simulations confirm that the in-plane magnetic field splits successfully when it reaches the first vertex [Fig. 2(e)]. To estimate the performance of the beam splitter, we calculate the splitting efficiency $\eta=1-\left(P_{\text {in }} / P_{\text {total }}\right)$, which compares the transmitted power inside the excluded region $\left(P_{\text {in }}\right)$ to the total power at the end facet of the splitter 


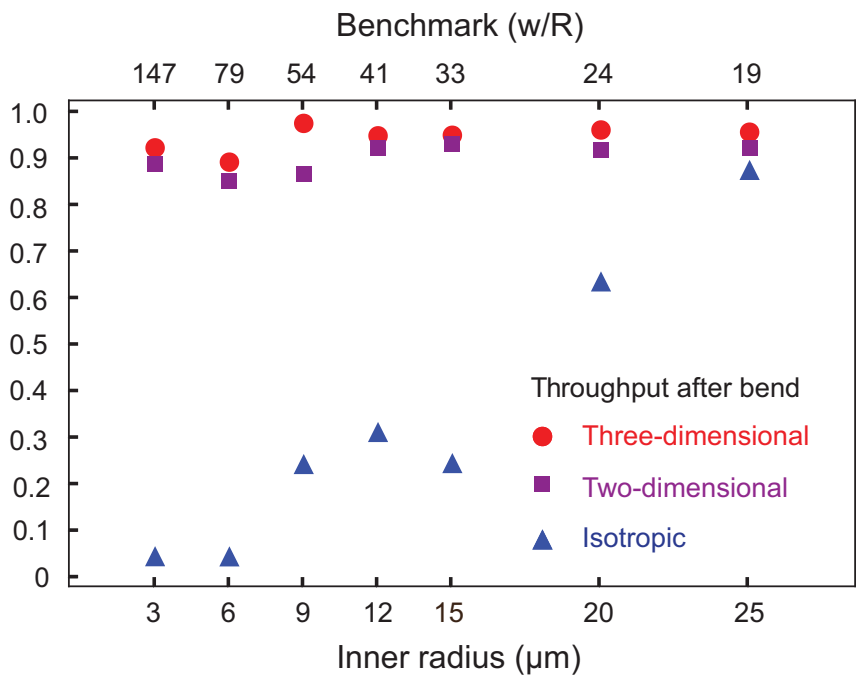

FIG. 3. The throughput of our beam bender is evaluated for seven outer radii $R$ at fixed beam width $w$, where increases in anisotropy are represented by increases in the benchmark $\frac{w}{R}$, as a way to demonstrate the effectiveness of our equivalence relations. In particular, we compare three implementations: the conventional design of transformation optics (red dots), our two-dimensional design (purple squares), and an isotropic implementation with appropriate thickness variation (blue triangles). The throughputs of our twodimensional metamaterial cores are impressive, lying close to those of the three-dimensional implementation while isotropic metamaterial cores cannot maintain their performance as inner radii approach the free space wavelength $\lambda=1.5 \mu \mathrm{m}$. Mesh convergence studies resulted in negligible error bars, although we suspect a systematic error since the three-dimensional implementations represent the ideal impedance-matched implementation corresponding to a theoretical throughput of unity.

$\left(P_{\text {total }}\right)$. The efficiency is adversely affected by the singularities of a Schwarz-Christoffel transformation, associated to vertices that impose vanishing rescalings $X=0$. Indeed, rescalings below a specific threshold (Fig. S2) lead to optically dilute cores which cannot confine light. This is expressed mathematically by an imaginary extinction coefficient $k_{1}$. Fortunately, subcritical rescalings_crucial to some applications such as the invisibility cloak [10-12] — can be eliminated by combining embedded transformations and truncations with appropriate global rescalings (Fig. S8). For example, our beam splitter easily attains a splitting efficiency of $81 \%$.

As a final example, we implement a two-dimensional lens based on the Möbius transformation [16]. Our design in Figs. 2(f)-2(g) requires realistic anisotropies and thickness variations and connects continuously to the untransformed waveguide behind the lens thanks to a suitable embedding (Fig. S10). Figure 2(g) confirms that the embedding does not affect the performance: the lens focuses in-plane magnetic fields extremely well, and more importantly, behaves in the

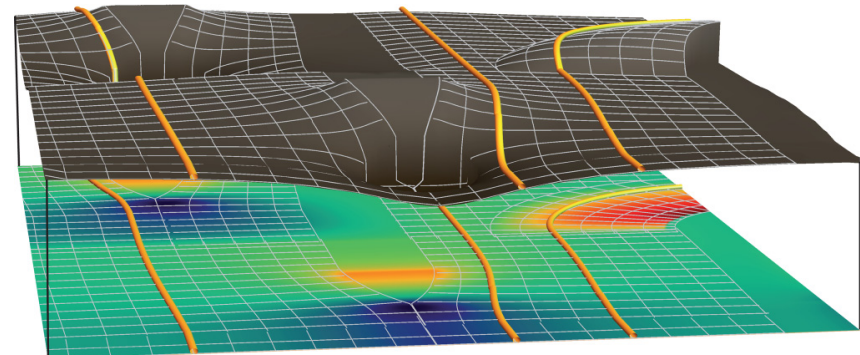

FIG. 4. Instead of manipulating individual light modes with numerous distinct fibers or waveguides, two-dimensional transformation optics manipulates an incident plane wave holistically by combining beam splitters, beam benders, and lenses in an integrated setup. Individual rays of an incident plane wave (yellow) are split and bent by the geometry-induced anisotropic material (visualized by surface coloring on the symmetry plane of the waveguide) and the thickness variation (visualized by floating surface representing the upper interface $z=\tilde{a}$ ). The deformed coordinate grid is projected both onto the waveguide's symmetry plane and the core-cladding interface.

same way as a three-dimensional transformation-optical lens (Fig. S11). Indeed, the spot size (determined by $\frac{1}{e}$ of the maximal amplitude) is as small as $1.6 \lambda_{0}$ with free space wavelength $\lambda_{0}$.

While this report is focused on a transformation optics framework applicable to two-dimensional waveguides, we want to briefly discuss the feasibility of the designs discussed above. Each of the three devices requires only dielectric response and no magnetic response. The $\epsilon_{\|}$component of the permittivity tensor is constant over the entire structure and can be chosen arbitrarily, and the anisotropies are fairly small. Such structures can be fabricated using 3D printing (direct laser writing) by using small subwavelength ellipsoids as constituent elements $[7,38,43]$, similar to how optical ground-plane cloaks have been realized [43].

The beam bender, beam splitter, and conformal lens constitute three independent examples that illustrate how our twodimensional equivalence relations allow for the manipulation of guided waves in coordinate-designed waveguides with realistic material parameters. Therefore, we envision that our results open up the geometrical toolbox of transformation optics for the manipulation of two-dimensional guided waves on multifunctional optical chips (Fig. 4).

\section{ACKNOWLEDGMENTS}

S.V. and V.G. acknowledge fellowships from the Research Foundation Flanders (FWO-Vlaanderen). Work at VUB was partially supported by the Research Council of the VUB and by the Interuniversity Attraction Poles program of the Belgian Science Policy Office, under Grant No. IAP P7-35 "photonics@be".
[1] M. Born and E. Wolf, Principles of Optics: Electromagnetic Theory of Propagation, Interference, and Diffraction of Light (Pergamon Press, New York, 1964).

[2] J. B. Pendry, Nat. Mater. 5, 599 (2006).
[3] N. Engheta and R. W. Ziolkowski, Metamaterials: Physics and Engineering Explorations (John Wiley \& Sons, New York, 2006).

[4] C. M. Soukoulis and M. Wegener, Nat. Photonics 5, 523 (2011). 
[5] A. V. Kildishev, A. Boltasseva, and V. M. Shalaev, Science 339, 1232009 (2013).

[6] N. I. Zheludev and Y. S. Kivshar, Nat. Mater. 11, 917 (2012).

[7] O. D. Lavrentovich, Proc. Natl. Acad. Sci. USA 108, 5143 (2011).

[8] J. D. Joannopoulos, S. G. Johnson, J. N. Winn, and R. D. Meade, Photonic Crystals: Molding the Flow of Light, 2nd ed. (Princeton University Press, New Jersey, 2011).

[9] J. B. Pendry, Y. Luo, and R. Zhao, Science 348, 521 (2015).

[10] J. B. Pendry, D. S. Schurig, and D. R. Smith, Science 312, 1780 (2006).

[11] U. Leonhardt, Science 312, 1777 (2006).

[12] B. Zhang, Light: Sci. Appl. 1, e32 (2012).

[13] A. Greenleaf, Y. Kurylev, U. Leonhardt, and G. Uhlmann, Proc. Natl. Acad. Sci. USA 109, 10169 (2012).

[14] T. Bückmann, M. Kadic, R. Schittny, and M. Wegener, Proc. Natl. Acad. Sci. USA 112, 4930 (2015).

[15] H. Chen, C. T. Chan, and P. Sheng, Nat. Mater. 9, 387 (2010).

[16] L. Xu and H. Chen, Nat. Photonics 9, 15 (2015).

[17] P. B. Catrysse and S. Fan, Adv. Mat. 25, 194 (2013).

[18] J. B. Pendry, A. Aubry, D. R. Smith, and S. A. Maier, Science 337, 549 (2012).

[19] V. Ginis, J. Danckaert, I. Veretennicoff, and P. Tassin, Phys. Rev. Lett. 113, 167402 (2014).

[20] F. Liu and J. Li, Phys. Rev. Lett. 114, 103902 (2015).

[21] D. K. Gramotnev and S. I. Bozhevolnyi, Nat. Photonics 4, 83 (2010).

[22] A. Grigorenko, M. Polini, and K. Novoselov, Nat. Photonics 6, 749 (2012).

[23] P. Genevet, D. Wintz, A. Ambrosio, A. She, R. Bianchard, and F. Capasso, Nat. Nanotechnol. 10, 804 (2015).

[24] A. Vakil and N. Engheta, Science 332, 1291 (2011).

[25] W. B. Lu, W. Zhu, H. J. Xu, Z. H. Ni, Z. G. Dong, and T. J. Cui, Opt. Express 21, 10475 (2013).

[26] Y. Liu, T. Zentgraf, G. Bartal, and X. Zhang, Nano Lett. 10, 1991 (2010).
[27] P. A. Huidobro, M. Nesterov, L. Martín-Moreno, and F. J. García-Vidal, Nano Lett. 10, 1985 (2010).

[28] M. Kadic, S. Guenneau, S. Enoch, P. A. Huidobro, L. Martín-Moreno, F. J. García-Vidal, J. Renger, and R. Quidant, Nanophotonics 1, 51 (2012).

[29] I. I. Smolyaninov, V. N. Smolyaninova, A. V. Kildishev, and V. M. Shalaev, Phys. Rev. Lett. 102, 213901 (2009).

[30] S. Xu, H. Xu, H. Gao, Y. Jiang, F. Yu, J. D. Joannopoulos, M. Soljacíc, H. Chen, H. Sun, and B. Zhang, Proc. Natl. Acad. Sci. USA 112, 7635 (2015).

[31] D. Sarid, Phys. Rev. Lett. 47, 1927 (1981).

[32] R. F. Oulton, V. J. Sorger, D. A. Genov, D. F. P. Pile, and X. Zhang, Nat. Photonics 2, 496 (2008).

[33] M. Law, D. J. Sirbuly, J. C. Johnson, J. Goldberger, R. J. Saykally, and P. Yang, Science 305, 1269 (2004).

[34] X. Fan and I. M. White, Nat. Photonics 5, 591 (2011).

[35] D. Van Thourhout and J. Roels, Nat. Photonics 4, 211 (2010).

[36] A. Snyder and J. Love, Optical Waveguide Theory (Springer Science \& Business Media, London, 1983).

[37] P. Tassin, T. Koschny, M. Kafesaki, and C. M. Soukoulis, Nat. Photonics 6, 259 (2012).

[38] S. J. Corbitt, M. Francoeur, and B. Raeymaekers, J. Quant. Spectrosc. Radiat. 158, 3 (2015).

[39] See Supplemental Material at http://link.aps.org/supplemental/ 10.1103/PhysRevB.93.085429 for more information about the derivation of our equivalence relations based on the Helmholtz equation and dispersion equation of a slab waveguide and indepth information concerning the applications, i.e., the beam bender, beam splitter, and conformal lens.

[40] R. Schinzinger and P. A. A. Laura, Conformal Mapping: Methods and Applications (Courier Dover Publications, New York, 2012).

[41] J. G. Laethem, Philos. Trans. R. Soc. London A 215, 439 (1915).

[42] D. A. B. Miller, J. Opt. Soc. Am. B 24, A1 (2007).

[43] T. Ergin, N. Stenger, P. Brenner, J. B. Pendry, and M. Wegener, Science 328, 337 (2010). 